\title{
La comprensión lectora inferencial mediante el uso del texto literario y cotidiano
}

Nubia Liliana Montero Yanquén *

Mary luz Cuervo López***

Artículo de investigación

Fecha de Recepción: 16 diciembre 2017.

Fecha de Aprobación: 19 mayo 2018.

\section{RESUMEN}

Este artículo muestra el avance preliminar de la implementación del proyecto titulado "La Comprensión Lectora Inferencial Mediante El Uso Del Texto Literario Y Cotidiano", desarrollado en la Institución Educativa Nuestra Señora de la Candelaria del municipio de Ráquira, con estudiantes de grado séptimo, estudio de carácter cualitativo descriptivo, hace énfasis en la explicación, análisis y resultados de la fase diagnóstica del proyecto, al respecto conviene decir que se tuvieron en cuenta los resultados del $\operatorname{ICSE}^{1}$ del año 2015, entiéndase texto literario como aquel que usa un lenguaje estético para llamar la atención del lector y como texto cotidiano aquel que es de uso común, como afiches, mensajes de texto, vallas, imágenes entre otros. Los resultados muestran que hay deficiencias con respecto a las competencias básicas de lenguaje tales como establecer relaciones de causa y efecto, contextualizar el significado de las palabras y del texto, reelaboración de texto escrito en una gráfica, predicción de resultados y consecuencias.

Palabras Clave: Lectura, Lectura inferencial, texto literario, texto cotidiano.
* I. E. Nuestra Señora de la Candelaria - sede Firita Peña arriba - Ráquira Boyacá.

lilianamontero2007@yahoo. es

** I. E. Nuestra Señora de la Candelaria - sede central

- Ráquira - Boyacá. malu18234@gmail.com

1 Índice Sintético de Calidad, informe por colegio 2015. Emitido por el MEN- synthetic quality index , 2015 school reportg

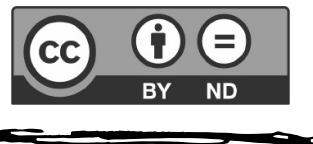




\section{INTRODUCCIÓN}

Según Solé (1992) la lectura es un proceso mental, en este proceso se deduce el significado de un texto, se interpreta su contenido, se comprende el mensaje, se realizan inferencias y cuestionamientos, eso es lo que se espera que logren los estudiantes en el proceso educativo, en el ejercicio académico diario y que les sirva además para la vida, para comprender e interactuar con su entorno.

Dubin et al. (2014) se refieren al proceso lector como un comportamiento cognitivo basado en los distintos tipos de conocimientos de la estructura cognitiva del sujeto. Dicha estructura la integran sus conocimientos, (lo que llamamos actualmente saberes previos), que constituyen a su vez lo que se ha llamado esquema (schema o schemata en inglés) en la memoria a largo plazo. Cuanto mejor sea la destreza lectora de una persona, más rápido podrá llevar a cabo el proceso. Durante la lectura el sujeto hace predicciones sobre el significado del texto, a medida que lo va reconstruyendo. Los resultados dependen de sus conocimientos y de su capacidad de razonamiento. Se trata de un proceso doblemente interactivo, en tanto que abarca la interacción del lector con sus conocimientos y con el texto.

Peronard, et al. (2002) asevera que lectura no es un proceso automático sino estratégico: en función del objetivo que se persiga, será el modo en el que se realice, los elementos en los cuales se ponga mayor atención, la cantidad de conocimientos previos que están en juego, el grado y el nivel de reestructuración del contenido, para hacerlo congruente a los esquemas mentales propios.

Teniendo en cuenta la realidad descrita, se busca hacer de la lectura inferencial, un ejercicio guiado de manera lúdica, en un escenario de aprendizaje con sentido crítico, activo, reflexivo y significativo, para que los conocimientos que adquieran los estudiantes fortalezcan el desarrollo de competencias lectoras, resolución pacífica de conflictos y aprendizajes que puedan ser puestos en práctica como herramientas útiles para la vida escolar y extraescolar.

Las organizaciones relacionadas con la educación a nivel mundial como son el programa de las Naciones Unidas para el Desarrollo (PNUD), el fondo de las Naciones Unidas para la Infancia (UNICEF), el Banco Mundial, Fondo de Población de las Naciones Unidas (FNUAP), adicionalmente los Institutos y Centros de la UNESCO especializados en educación se han interesado por orientar los procesos educativos hacia el mejoramiento de las prácticas de aula y el fortalecimiento de la lectura, como medio de construcción del conocimiento; dentro del contexto escolar, la lectura hace parte del escenario educativo como eje generador de conocimiento, según estudios, el proceso lector es una práctica poco implementada por los estudiantes de forma autónoma en cada uno de los niveles educativos, por lo tanto, se ven afectados los procesos 
de aprendizaje en todas las áreas del conocimiento y, en general, en toda su formación integral, es por eso que se hace énfasis en el estudio del desarrollo de habilidades del proceso de lectura inferencial por medio del texto literario y cotidiano.
El texto cotidiano comprende textos de la vida cotidiana y las relaciones sociales: son textos que se emplean en un contexto familiar y social, el colegio: cartas, avisos, notas, normas, instrucciones, entre otros, entre los textos de la vida cotidiana y las relaciones sociales, se pueden distinguir los siguientes, ver figura 1.

\begin{tabular}{|l|l|}
\hline \multicolumn{2}{|c|}{ TIPOS DE TEXTO COTIDIANO } \\
\hline TIPO & \multicolumn{1}{c|}{ DESCRIPCION } \\
\hline NOTAS & $\begin{array}{l}\text { Las notas responden a distintas finalidades: informar, rogar, agradecer, disculparse, comentar. } \\
\text { También hay notas públicas y privadas. }\end{array}$ \\
\hline AVISOS & $\begin{array}{l}\text { Son escritos que tienen la finalidad de alertar o prevenir. Pueden aparecer en lugares públicos } \\
\text { o en lugares privados Algunos, tiene un significado más general, o pueden ser más familiares. }\end{array}$ \\
\hline CARTAS & $\begin{array}{l}\text { La carta es uno de las formas de comunicación más cercanas y cotidianas, aunque la forma } \\
\text { de escribirlas actualmente ha cambiado, el encanto de la misiva escrita se ha perdido con } \\
\text { el desarrollo de las nuevas tecnologías. Ahora tenemos opciones mucho más rápidas y } \\
\text { cómodas como el e-mail, el mensaje a móviles, los WhatsApp. }\end{array}$ \\
\hline NORMAS & $\begin{array}{l}\text { Son reglas que establecen cómo ha de ser o cómo debe hacerse algo. Hay normas que se } \\
\text { recogen en reglamentos y leyes y son de cumplimiento obligatorio. Por ejemplo, hay que } \\
\text { cumplir las normas establecidas en el manual de convivencia. }\end{array}$ \\
\hline INSTRUCCIONES & $\begin{array}{l}\text { Son indicaciones que orientan acerca de la manera de hacer o usar algo. No son de obligado } \\
\text { cumplimiento como las normas. }\end{array}$ \\
\hline
\end{tabular}

Figura 1. Tipos de texto cotidiano - creación propia.

Como texto literario se denomina todo aquel en el que se reconocen las características propias de algún género literario, como la narrativa, la poesía, el teatro o el ensayo. Desde el punto de vista de la Lingüística, el texto literario es aquel donde predomina la función poética del lenguaje, es decir, donde la composición del texto atiende fundamentalmente a la forma en sí del lenguaje, a su belleza y a su capacidad para recrearnos mundos imaginarios.

Algunas de las características de los textos literarios son la libertad creativa plena en cuanto a la composición: la forma, el estilo y el tono del texto (puede ser triste, alegre, amargo, irónico, festivo); en el empleo de figuras literarias, como la metáfora, la metonimia o el símil, para enriquecer el texto y avivar la imaginación del lector; en el manejo de los recursos discursivos como la narración, la descripción, el diálogo, la exposición o la argumentación, para conducir el texto; así como la naturaleza ficticia de que está hecho el texto literario. (Eagleton, 2016). 
En el caso de la lectura de textos literarios esta actividad interactiva presenta una serie de particularidades por la relación que se establece entre quien lee y el texto leído, en tanto que éste, no es sino en términos de Umberto Eco (1991) un "mundo posible”. Sullé (1982) p253, afirma que "Se trata de un universo en el que según no sólo interactúan los personajes de los que el texto habla, sino también aquellos que hablan en el texto: el enunciador y el enunciatario.”, tal y como indica Foucault "el lector cuando lee un texto literario se convierte en narratario, "alguien a quien el narrador dirige sus palabras"

El texto literario, tal y como Lotman (2000) afirma tiene tres funciones básicas: una función comunicativa, otra semiótica, generativa o creadora de significados y otra simbolizadora, que convierten la lectura del texto literario en reflejo de la cultura y motor de la interculturalidad. Al igual que Denyer (1999) creemos que la lectura que hemos de promover y más en el caso de estudiantes del nivel de básica secundaria debe respaldarse con un buen conocimiento del código lingüístico, como lo es la inferencialidad. Tal y cómo explica Sanz (1997), se trata de que el aprendiente haga suyos los espacios de connotación y sea capaz de leer lo que está escrito y lo que no lo está.

El texto literario es una fuente para el aprendizaje del código lingüístico, en tanto que leer implica la habilidad para descodificar e interpretar un discurso en contexto, contribuye asimismo a la construcción de la competencia cultural en tanto que refleja la realidad y el imaginario de los hablantes de una lengua en un momento determinado. A través de la lectura de textos literarios promovemos el desarrollo de la competencia intercultural ya que el texto literario contribuye a la construcción de un contexto que se ve ampliado con la lectura de otros textos literarios, también se desarrolla la competencia literaria de los estudiantes cuya existencia queda justificada en tanto que implica la puesta en práctica de unos procesos y micro habilidades que se ponen en marcha a través de los actos de lectura y escritura.

La lectura de textos literarios se establece como un instrumento clave en la construcción de la competencia comunicativa de los estudiantes y del fortalecimiento de la comprensión lectora inferencial ya que además contribuye a la construcción de un contexto que permite leer más y más textos.

Los educandos, cuando están inmersos en el proceso educativo formal de básica secundaria, se enfrentan a nuevas formas de alcanzar competencias lectoras, interpretación y acercamiento hacia los textos. El contexto académico escolar plantea diversas representaciones de contacto con la lectura, por tal razón deben desarrollar niveles óptimos en los procesos de comprensión lectora que los lleven a responder a tales exigencias.

Ante esta problemática, la UNESCO (2015), dentro del Segundo Estudio Regional Comparativo y Explicativo 
(SERCE) del año 2010, en el cual se pretende brindar orientaciones a los docentes para que se les ayude a mejorar sus prácticas de aula en torno a la enseñanza de la lectura, desde el reconocimiento de diferentes tipos de textos (verbales y no verbales). Por lo cual se puede evidenciar que la lectura como medio de generación del conocimiento y movilizadora de aprendizajes significativos, ha sido una preocupación constante que se ve igualmente reflejada en el quehacer docente.

Este artículo pretende dar cuenta del estado inicial de diagnóstico de la investigación " $\mathrm{La}$ comprensión lectora inferencial mediante el uso del texto literario y cotidiano" desarrollada con los estudiantes de grado séptimo de la Institución Educativa Nuestra Señora de la Candelaria de Ráquira, no responde al objetivo total del proyecto, se describe la forma como se desarrolló, aplicó y analizó la fase inicial del mismo para identificar el nivel de lectura inferencial evidenciado en los estudiantes.

Al reflexionar acerca del quehacer diario en La Institución Educativa Nuestra Señora de la Candelaria de Ráquira y basados en las prácticas educativas desarrolladas con respecto a la lectura, se encuentra que en los últimos años se han desarrollado diversos proyectos tendientes a mejorar los hábitos lectores en los estudiantes, como son; lectura desde las Tics, lectura de obras literarias, jugando con ortografía, leer es mi cuento, semana de la lectura dirigida, semana de la lectura interactiva y lectura de textos discontinuos.

Mendoza (2003) ha llevado a cabo una conceptualización de la lectura afirmando que ésta ya no se considera como una descodificación basada en el texto, sino una interacción entre el lector y el texto, ya que "descodificar no es leer, pero necesitamos descodificar para comprender lo que leemos" a esto se suma la importancia que tiene el proceso mental que debe haber después de la descodificación, el análisis y la inferencia, factores claves en la comprensión lectora, aspectos que tiene en cuenta Cassany (2006) cuando indica que "la lectura es un acto de comprensión, pero, para ello es necesario desarrollar varias destrezas mentales o procesos cognitivos, tales como: anticipar lo que dirá el escrito; aportar los conocimientos previos; hacer hipótesis; elaborar inferencias para comprender lo que solo se sugiere, etc." Esto nos muestra que el acto de leer es complejo, lo cual lo hace objeto de investigación y análisis en las aulas de clase para describir como se está llevando a cabo este proceso, identificar las problemáticas y plantear una intervención pedagógica que aporte al mejoramiento de la comprensión lectora inferencial, que es aquella que pretende que el estudiante extraiga información explícita o implícita de un texto.

Muchos lectores tienen dificultades para responder adecuadamente a preguntas referentes al contenido de textos previamente leídos McNamara (2004); esto se debe a diversos factores
Mendoza, (2003) ha llevado a cabo una conceptualización de la lectura afirmando que ésta ya no se considera como una descodificación basada en el texto, sino una interacción entre el lector y el texto, ya que "descodificar no es leer, pero necesitamos descodificar para comprender lo que leemos" 
que están relacionados con uno o varios de los procesos de lectura como la decodificación y la comprensión, o a dificultades del pensamiento relacionadas con estos procesos, Cisneros (2010).

De acuerdo con Solé (1992) la lectura inferencial es una estrategia, en la cual el lector, a partir de los datos que extrae del texto, elabora suposiciones susceptibles de verificación o sustentación. Inferir es leer entre líneas, las inferencias se pueden trabajar a través de textos escogidos para ello, pero también se pueden hacer prácticas exclusivas para que los niños o jóvenes aprendan a buscar las pistas o simplemente a fijarse en estas, para detectarlas.

\section{METODOLOGIA}

Con el propósitoderesponderalobjetivo principal, se hizo necesario asumir una metodología de investigación orientada por un enfoque cualitativo con un diseño de tipo descriptivo, se adopta esta metodología teniendo en cuenta lo que dice Sampieri (2008) pP.8, "la investigación cualitativa proporciona profundidad a los datos, dispersión, riqueza interpretativa, contextualización del ambiente o entono, detalles y experiencias Únicas. También aporta un punto de vista "fresco, natural y completo" de los fenómenos, así como flexibilidad. La investigación fue descriptiva, ya que se registraron, analizaron e interpretaron los datos que arrojó la prueba diagnóstica y cualitativa, en ella se destacan las características o rasgos de la situación, y su función principal es la capacidad para seleccionar las características fundamentales del objeto de estudio.

Esta investigación tiene su origen en el análisis de resultados del Índice Sintético de Calidad de La Institución Educativa Nuestra Señora De La Candelaria en donde se evidencia el bajo desempeño en la competencia lectora de los estudiantes de educación media, el informe del año 2015 en donde se afirma a propósito del componente semántico de la competencia lectora que "el 48\% de los estudiantes no selecciona los mecanismos que aseguran la articulación sucesiva de las ideas en un texto (presentación, continuación, transición, digresión, enumeración, cierre o conclusión) atendiendo al tema central, esto muestra que el estudiante no logra elegir los elementos lingüísticos que ayudan a ordenar y estructural el texto.

En relación al componente sintáctico el informe dice que "El $60 \%$ de los estudiantes no identifica la estructura explícita del texto (silueta textual).” Es decir que se dificulta la acción de asemejar la correferencialidad, el estudiante no logra Identificar la función de marcas lingüísticas de cohesión local (entre oraciones y párrafos) y también se dificulta el hecho de reconocer algunas estrategias propias de cada tipo textual como ubicar el texto dentro de una tipología o taxonomía (por el uso o función).

Con respecto al componente semántico afirma que "El 46\% de los estudiantes no recupera información implícita en el contenido del texto." Lo que 
significa una dificultad para Identificar el sentido que tienen algunos códigos no verbales en situaciones de comunicación cotidianas, también presentan dificultad para reconocer la presencia de argumentos de un texto, la secuencia de acciones o acciones proceso (hechos, eventos, pasos, momentos, etapas e instrucciones).

En cuanto al componente pragmático se evidencia según los resultados obtenidos que "El $42 \%$ de los estudiantes no evalúa información explícita o implícita de la situación de comunicación." Significa que el estudiante no logra Identificar intenciones, propósitos y perspectivas, tampoco se le facilita identificar la función social de algunos textos de circulación cotidiana, adicionalmente el informe también muestra que "El $53 \%$ de los estudiantes no identifica la estructura implícita del texto".

Partiendo de este panorama se planeó una prueba inicial, tipo taller, con tareas de lectura, en la que participaron 34 niños, 14 niños y 20 niñas, todos pertenecientes al nivel socioeconómico bajo (estratos 1-2). En la prueba de test diagnóstico se aplicaron ejercicios de pensamiento inferencial y de comprensión de lectura, utilizando textos literarios y cotidianos, donde se realizó la exploración de habilidades de lectura.

El instrumento, un taller diagnóstico, fue planteado teniendo en cuenta que el taller es una estrategia didáctica para mejorar la comprensión lectora, según Giraldo et, al (2008) "el punto de partida para la planificación del taller es identificar las necesidades que se buscan responder, las cuales deben haberse traducido a unos objetivos que son determinados por el docente o agente educativo" en este caso el taller fue diseñado por las investigadoras, y se tomó como referente las pruebas SABER que han sido aplicadas por el Ministerio de Educación Nacional. El instrumento de evaluación del nivel de comprensión está estructurado por un conjunto de doce tareas de lectura (ver figura 1).

\begin{tabular}{|l|l|l||}
\hline \multicolumn{3}{|c|}{ TAREAS DE LECTURA TEST DIAGNÓSTICO. } \\
\hline Punto $\mathrm{N}^{\circ}$ & \multicolumn{1}{|c|}{ Tarea de lectura } & \multicolumn{1}{c|}{ Descripción } \\
\hline \multirow{1}{*}{1.} & $\begin{array}{l}\text { Leer la letra de la canción "despacito" con } \\
\text { algunas palabras subrayadas. }\end{array}$ & $\begin{array}{l}\text { Se pretende evaluar si el estudiante logra identificar de } \\
\text { qué manera se está usando esa palabra en la oración, } \\
\text { para este proceso de comprensión lectora inferencial, } \\
\text { Cassany (2006) nos presenta cuatro estrategias: la } \\
\text { primera, atender la morfología. Es decir, fijarnos } \\
\text { en su lexema (raíz). Y afijos (prefijos y sufijos), en } \\
\text { segundo lugar, utilizar el diccionario para reconocer }\end{array}$ \\
$\begin{array}{l}\text { A partir de allí se pide elaborar un diccionario } \\
\text { con 10 de las palabras, el significado debe } \\
\text { buscarse en la letra de la canción de acuerdo } \\
\text { con su contexto. }\end{array}$ & $\begin{array}{l}\text { en palabras de tatros idiomas, especialmente cuando } \\
\text { necesitamos reconocer el significado de palabras de } \\
\text { ámbitos especializados y, por último, observar en el } \\
\text { valor del contexto. Es decir, estudiar el resto de las } \\
\text { palabras del fragmento en el que aparece el vocablo } \\
\text { desconocido. }\end{array}$ \\
\hline
\end{tabular}




\begin{tabular}{|c|c|c|}
\hline 2 & $\begin{array}{l}\text { Buscar en la letra de la canción las palabras } \\
\text { resaltadas, y ordenarlas alfabéticamente en su } \\
\text { diccionario, de la misma forma ellos escriben } \\
\text { su significado y deben complementar con una } \\
\text { ilustración. }\end{array}$ & $\begin{array}{l}\text { se pretende con esta actividad seguir las indicaciones } \\
\text { dadas por los lineamientos de lenguaje }{ }^{2} \text { en donde } \\
\text { se afirma que "Las actividades de ordenamiento de } \\
\text { frases suponen un proceso de reescritura mediante el } \\
\text { cual determinadas palabras adquieren un significado } \\
\text { al estar ordenadas de una forma determinada dentro } \\
\text { de una frase, que adquiere un significado global y } \\
\text { una correcta estructura sintáctica." }\end{array}$ \\
\hline $\begin{array}{l}3.1 \\
3.2 \\
3.3\end{array}$ & $\begin{array}{l}\text { Realizar la lectura del texto de } 10 \text { renglones } \\
\text { titulado "El Capitán América" a partir de se } \\
\text { pide a los estudiantes desarrollar cuatro tareas: } \\
\text { a. En la línea de tiempo marca los hechos de } \\
\text { surgimiento del Capitán América. } \\
\text { b. Enumera las cualidades para las cuales fue } \\
\text { creado este súper héroe. } \\
\text { c. Escribe dos relaciones entre el Capitán } \\
\text { América y Marvel Comics. } \\
\text { d. Responde: ¿En nuestro país a quienes } \\
\text { llamamos justicieros? }\end{array}$ & $\begin{array}{l}\text { Aprendizajes que se evalúan en las pruebas saber } \\
\text { en los componentes pragmático, sintáctico y } \\
\text { semántico. Se busca que el estudiante, mediante la } \\
\text { representación gráfica de información e ideas, aclare } \\
\text { sus pensamientos, refuerce su comprensión, integre } \\
\text { nuevo conocimiento (organizando, procesando y } \\
\text { priorizando información nueva o ya conocida) e } \\
\text { identifique conceptos erróneos. Adicionalmente } \\
\text { se mide el nivel de inferencia lectora al pedirles } \\
\text { que enumeren cualidades, que escriban relaciones } \\
\text { percibidas entre los personajes de la lectura y que } \\
\text { identifiquen lo real de lo imaginario relacionándolo } \\
\text { con el entorno real. }\end{array}$ \\
\hline 4.1 & $\begin{array}{l}\text { Observar una imagen en donde se encuentran } \\
10 \text { súper héroes, leer el texto de } 12 \text { renglones } \\
\text { que complementa, en donde se describen } \\
\text { las cualidades y características del Capitán } \\
\text { América. } \\
\text { De acuerdo a la lectura selecciona la bandera } \\
\text { del país que El Capitán América lleva en su } \\
\text { ropa. Escoger una de veinte posibilidades. }\end{array}$ & $\begin{array}{l}\text { En el cuarto punto se pide relacionar la información } \\
\text { de un texto identificando sus características } \\
\text { específicas, este aspecto se desarrolló teniendo en } \\
\text { cuenta la importancia de las 'teorías implícitas' } \\
\text { entendidas como un tipo de idea cognitiva, } \\
\text { inconsciente, latente e implícita, que ejercen una } \\
\text { enorme influencia en las conductas, decisiones y } \\
\text { acciones o respuestas de los 'sujetos' a determinados } \\
\text { problemas del conocimiento. Smith, et, al (2009) }\end{array}$ \\
\hline 4.2 & $\begin{array}{l}\text { Dibujar el traje del personaje que le llame la } \\
\text { atención de los Vengadores y relacionarlo con } \\
\text { alguna bandera. }\end{array}$ & $\begin{array}{l}\text { Se busca verificar si se logró la comprensión o no } \\
\text { de la lectura indicando que ilustre el contenido de } \\
\text { manera gráfica. }\end{array}$ \\
\hline 5. & $\begin{array}{l}\text { Lea las características los siguientes textos } \\
\text { (El Nissan Skyline, y el Toyota Supra) y } \\
\text { clasifique la información en un cuadro que } \\
\text { se presenta. }\end{array}$ & $\begin{array}{l}\text { Se pretende mediante este ejercicio evaluar la } \\
\text { capacidad lectora del estudiante mediante la } \\
\text { capacidad de agrupar los elementos de información } \\
\text { de acuerdo a atributos o propiedades comunes entre } \\
\text { ellos. }\end{array}$ \\
\hline
\end{tabular}

Figura 2. descripción de las tareas de lectura del taller diagnóstico. - Creación propia

\section{RESULTADOS}

Para la obtención de los resultados de la fase diagnóstica se tabuló cada pregunta teniendo en cuenta una rúbrica de evaluación de creación propia, en donde se evalúa mediante el uso de criterios de lectura inferencial planteados por el MEN, criterios que deben poseer los estudiantes de grado séptimo en el área de lenguaje, las respuestas se clasifican en resultados con un carácter Alto, entre 22 - 32 estudiantes, aceptable, entre 12 21 estudiantes, no aceptable, entre 0 - 11 estudiantes. 


\begin{tabular}{|c|c|c|c|}
\hline \multicolumn{4}{|c|}{$\begin{array}{l}\text { UNIVERSIDAD PEDAGÓGICA Y TECNOLÓGICA DE COLOMBIA } \\
\text { MAESTRÍA EN EDUCACIÓN MODALIDAD PROFUNDIZACIÓN } \\
\text { RUBRICA DE EVALUACIÓN } \\
\text { TALLER DIAGNOSTICO. }\end{array}$} \\
\hline CRITERIOS & ALTO & ACEPTABLE & $\begin{array}{c}\text { NO ACEP } \\
\text { TABLE }\end{array}$ \\
\hline Contextualizar el significado de las palabras y del texto. & $20 \%$ & $35 \%$ & $45 \%$ \\
\hline Reconocimiento de un hecho. & $30 \%$ & $28 \%$ & $42 \%$ \\
\hline Establece relaciones de causa y efecto. & $18 \%$ & $27 \%$ & $55 \%$ \\
\hline $\begin{array}{l}\text { 4. Establecer relaciones de causalidad entre las distintas } \\
\text { partes del texto. }\end{array}$ & $32 \%$ & $40 \%$ & $28 \%$ \\
\hline $\begin{array}{l}\text { Discriminar lo real de lo imaginario. } \\
\text { Deducción de conclusiones }\end{array}$ & $15 \%$ & $\begin{array}{l}80 \% \\
43 \%\end{array}$ & $\begin{array}{l}20 \% \\
42 \%\end{array}$ \\
\hline Narra historias cuyas ideas guardan relación entre sí & $50 \%$ & $25 \%$ & $25 \%$ \\
\hline Predicción de resultados y consecuencias. & $33 \%$ & $27 \%$ & $40 \%$ \\
\hline Explica con sus propias palabras los mensajes que lee & $37 \%$ & $17 \%$ & $46 \%$ \\
\hline Reelaboración del texto escrito en una gráfica. & & $45 \%$ & $55 \%$ \\
\hline $\begin{array}{l}11 . \quad \text { Deduce adecuadamente situaciones o acciones posibles a } \\
\text { sucederse. }\end{array}$ & $25 \%$ & $34 \%$ & $41 \%$ \\
\hline
\end{tabular}

Teniendo en cuenta lo anterior, Con respecto al primer criterio: Contextualizar el significado de las palabras y del texto, el 45\% de los estudiantes contestaron de manera no aceptable, no explican con sus propias palabras los mensajes que leen, se puede evidenciar en las respuestas dadas en el punto 2 de la guía 1 , por ejemplo, se le pregunta a un estudiante que identifique teniendo en cuenta la lectura las cualidades para las cuales fue creado el súper héroe, y ellos explican lo siguiente: "es un soldado, lucha contra el mal, protege a los bondadosos", sin embargo hay varios pliegos sin respuesta, lo cual evidencia que sí hay un nivel de comprensión, pero el resultado es bajo, hay muchas respuestas copiadas, varios pliegos tienen las mismas respuestas lo que tam- bién deja ver, el bajo nivel de lectura inferencial de los estudiantes.

En el criterio reconocimiento de un hecho: narra historias que guardan relación entre sí los estudiantes obtuvieron un $42 \%$ respuesta no aceptable, siendo el mayor porcentaje, esto se demuestra en la pregunta 4,2 del taller número uno, en donde se les solicita a los estudiantes que dibujen el traje del personaje que les llama mayormente la atención y que lo relacione con alguna bandera de las que aparecen en la lista, la mayoría realizaron el dibujo de acuerdo a la historia, pero un gran número no lo relacionaron con alguna bandera de la lista, se presenta de manera incompleta las respuestas lo que determina el bajo nivel de dominio de la competencia inferencial. 
Los estudiantes narran historias cuyas ideas guardan relación entre sí, estableciendo relaciones de causa y efecto, un $55 \%$ de estudiantes obtuvieron un resultado no aceptable, se evidencia esa característica en la pregunta número 4 en donde debían elaborar el dibujo del traje del personaje que le llamó la atención y además relacionarlo con alguna bandera, realizaron el dibujo de un súper héroe, lo cual evidencia que pueden escribir o plasmar una historia que guarda relación entre sí de acuerdo con el texto leído pero muchos no lo relacionaron con ninguna bandera.

Con respecto al criterio discrimina lo real de lo imaginario, el 80\% contestó de manera acertada la pregunta relacionada con respecto a escribir claramente a quien le llamamos súper héroe en Colombia, el otro 20\% confundió lo real con lo imaginario y escribió aspectos que no se ajustan a la realidad.

Para el criterio deducción de conclusiones, el 42\% respondió de manera aceptable y el 43\% de manera no aceptable, la evidencia se encuentra en la pregunta 4.1 del taller en donde de acuerdo a la lectura se debe identificar y señalar dentro de 20 posibilidades, la bandera del país que el súper héroe lleva en su ropa, las respuestas fueron casi equitativas entre los que acertaron y los que fallaron identificando la bandera.

En el criterio que hace referencia a narrar historias cuyas ideas guardan relación entre sí, los porcentajes obtenidos en desempeño aceptable y no aceptable son del 25\% cada uno y el mayor resultado fue de $50 \%$ en el promedio alto. Lo cual quiere decir que la mayoría de estudiantes en la pregunta número 5 en donde se indica que se debe leer las características de dos textos y clasificarlos en el cuadro que se dispone, logran plasmar la secuencia lógica del texto, factor que se convierte en fortaleza para la lectura inferencial.

En el aspecto evaluado predicción de resultados y consecuencias, el mayor porcentaje fue $40 \%$ en nivel bajo, se presenta dificultad, por ejemplo, en la pregunta 2.3 en donde se pide al estudiante que escriba dos relaciones entre el súper héroe y una entidad, muchos estudiantes no lograron identificar ese tipo de resultado a partir del análisis. Escribieron respuestas no aceptables, por ejemplo: "creador de la paz", "creador de los súper héroes", "parece común”, "creación del capitán América” o dejaron en blanco el espacio de la respuesta, lo que evidencia gran dificultad de lectura inferencial.

En el criterio Explica con sus propias palabras los mensajes que lee, presentó $46 \%$ de resultado no aceptable, lo que significa que no logran reconstruir el mensaje de un texto, se dificulta realizar inferencia y explicar con sus propias palabras lo que un emisor quiere decir en un texto. Es indiscutible ya que se evidencia en el punto número 2.4 cuando se les pregunta a los estudiantes que expliquen con sus propias palabras a que llamamos justicieros en nuestro país y las respuestas son; "Batman", "los que hacen los ejercicios", "a los luchadores" etc, esto permite 
determinar el grado de dificultad que presentan los estudiantes para explicar con sus propios términos el contenido implícito de un texto.

Mientras que para reelaborar un texto escrito en una gráfica los resultados fueron $44 \%$ aceptables y 55\% no aceptables, en este caso para demostrar la comprensión el estudiante debe inferir información de un texto y ordenarlo en una tabla dándole sentido según lo leído, encontrándose que hay un nivel de dificultad en este proceso.

Al evaluar la deducción adecuada de situaciones o acciones posibles a sucederse se encontró que el porcentaje más alto es $41 \%$ en nivel no aceptable, el estudiante debería identificar la estructura de un texto reconocer y separar algunos elementos significativos plasmándolos en una línea del tiempo, pregunta este ejercicio generó mucha confusión y dificultad en los estudiantes porque no relacionaban el año con el hecho correspondiente, muchos de ellos solo escribieron las fechas que aparecían en el texto, otros escribieron textualmente cuando apareció el capitán América. lo que indica que no hay sentido de relación textual.

Se evidencia que existe una problemática en la institución educativa con los estudiantes de grado séptimo en cuanto a comprensión lectora inferencial se refiere, la mayoría de los resultados de los criterios evaluados están en resultado no aceptable, lo que requiere una intervención para la solución de este problema.

\section{CONSIDERACIONES FINALES}

Para fortalecer aspectos de la comprensión lectora, y que fueron detectados con el diagnostico, se trabaja con un proyecto que pretende diseñar y aplicar una propuesta pedagógica que promueva el mejoramiento de la competencia lectora inferencial de los estudiantes del grado séptimo, demostrar el progreso del aprendizaje de la lectura inferencial evidenciado en los estudiantes participantes de la investigación $y$ adicionalmente evaluar la efectividad de la propuesta pedagógica de lectura inferencial a través del uso del texto literario y cotidiano frente a los progresos de comprensión inferencial.

Para alcanzar estas pretensiones se escogerá la muestra de trabajo, que serán los 15 estudiantes con más bajo desempeño en la prueba inicial, a partir de esto se crearán talleres de comprensión lectora haciendo énfasis en el uso de los textos cotidianos y literarios, teniendo en cuenta la edad de los estudiantes, los estándares básicos de competencias y los derechos básicos de aprendizaje propuestos por el ministerio de educación nacional. Se usarán distintos tipos de ejercicios de lectura como son análisis de imágenes, descripción de una escena, completar textos, ordenar párrafos, determinar mensajes, definir intención comunicativa de un texto, identificar similitudes y diferencias, escribir características según lectura de imágenes, entre otros, propuestos de manera agradable pensados y planeados
Se evidencia que existe una problemática en la institución educativa con los estudiantes de grado séptimo en cuanto a comprensión lectora inferencial se refiere, la mayoría de los resultados de los criterios evaluados están en resultado no aceptable, lo que requiere una intervención para la solución de este problema. 
con intencionalidad para que permitan suficientes para medir el grado mejora desarrollar competencias de lectura del dominio de habilidades lectoras de inferencial y así tener elementos los estudiantes.

\section{REFERENCIAS}

Cassany (2006). Tras las líneas: sobre la lectura contemporánea. Barcelona: Anagrama.

Denyer, (1999). La lectura: una destreza pragmática y cognitivamente activa. Universidad Antonio de Nebrija.

Dubin, M. (2014). Descolonizar la lengua y la literatura. El Toldo de Astier, 68

Eagleton, (2016). Una introducción a la teoría literaria. Fondo de Cultura económica.

Eco, H. (1981). La cooperación interpretativa en el texto narrativo. Barcelona: Lumen.

Lotman, et, al, (2000). La semiosfera: Semiótica de las artes y de la cultura. III, 3. Universidad de Valencia.

McNamara, D. (2004). Aprender del texto: Efectos de la estructura textual y las estrategias del lector. Revista Signos, 37 (55), 1-12.

Mendoza, (2003). Didáctica de la Lengua y la Literatura. Barcelona: Prentice Hall.

Peronard, M., Velásquez, M., Crespo, N., \& Viramonte, M. (2002). Conocimiento metacognitivo del lenguaje escrito: Instrumento de medida y fundamentación teórica. Infancia y aprendizaje, 25 (2), 131-145, https://doi. org/10.1174/021037002317417787

Sampieri, et, al. (1998). Metodología de la investigación (Vol. 1). México: Mcgraw-hill.

Sanz, S. (1997). Experto en enseñanza del español como lengua extranjera: Principios metodológicos de los enfoques comunicativos. Fundación Antonio de Nebrija.

Solé, I. (1992). Estrategias de lectura (Vol. 137). Graó.

Sullé, D. (1982). Teoría de la novela. Antología textos del siglo XX, Barcelona, España: Crítica. 